\title{
ORLICZ SPACES OF FINITELY ADDITIVE SET FUNCTIONS, LINEAR OPERATORS, AND MARTINGALES ${ }^{1}$
}

BY J. J. UHL

Communicated by A. Zygmund, July 19, 1966

The purpose of this note is to announce some properties and applications of Orlicz spaces of finitely additive set functions, the $V^{\Phi}$ spaces. The $V^{\Phi}$ spaces are natural generalizations of the $V^{p}$ spaces (Bochner [2] and Leader [6]).

1. The $V^{\Phi}(\mathfrak{X})$ spaces. Throughout this note $\Omega$ is a point set, $\Sigma$ a field of subsets of $\Omega, \mu$ a finitely additive extended real valued nonnegative set function defined on $\Sigma$; and $\Sigma_{0} \subset \Sigma$ is the ring of sets of finite $\mu$-measure. A partition $\pi$ is a finite disjoint collection $\left\{E_{n}\right\} \subset \Sigma_{0}$. The partitions are partially ordered by defining $\pi_{1} \leqq \pi_{2}$ whenever each $E_{n} \in \pi_{1}$ is a union of members of $\pi_{2}$. $\mathfrak{X}$ and $\mathfrak{V}$ are Banach (or $B$-) spaces with conjugate spaces $\mathfrak{X}^{*}$ and $\mathfrak{Y}^{*}$ respectively. $\Phi$ is a (nontrivial) Young's function with complementary function $\Psi$.

Definition. $V^{\Phi}(\Omega, \Sigma, \mu, \mathfrak{X})=\left(V^{\Phi}(\mathfrak{X})\right)$ consists of all finitely additive $\mu$-continuous $\mathfrak{X}$-valued set functions $F$ on $\Sigma_{0}$ such that for some $k>0$,

$$
I_{\Phi}(F / k)=\sup _{\pi} \sum_{\pi} \Phi\left(\frac{\left\|F\left(E_{n}\right)\right\|}{k \mu\left(E_{n}\right)}\right) \mu\left(E_{n}\right) \leqq 1,
$$

where the supremum is taken over all partitions $\pi=\left\{E_{n}\right\}$ and the convention $0 / 0=0$ is observed.

$V^{\Phi}(\mathfrak{X})$ becomes a $B$-space under each of the equivalent norms

or

$$
N_{\Phi}(F)=\inf \left\{k>0: I_{\Phi}(F / k) \leqq 1\right\}
$$

$$
\|F\|_{\Phi}=\sup \left\{\sup _{\pi} \sum_{\pi} \frac{\left\|F\left(E_{v}\right)\right\|\left\|G\left(E_{v}\right)\right\|}{\mu\left(E_{v}\right)}: G \in V^{\Psi}\left(\mathfrak{X}^{*}\right), N_{\Psi}(G) \leqq 1\right\} .
$$

Using the integration procedure of $[4$, Chap. III $]$, one can define the (possibly incomplete) Orlicz spaces $L^{\Phi}(\Omega, \Sigma, \mu, \mathfrak{X})\left(=L^{\Phi}(\mathfrak{X})\right)$ of totally $\mu$-measurable $\mathfrak{X}$ valued functions $f$ satisfying $\int_{\Omega} \Phi(\|f\| / k) d \mu$ $\leqq 1$ for some $k>0$. $L^{\Phi}(\mathfrak{X})$ becomes a normed linear space under either of the two equivalent norms $N_{\Phi}(f)=\inf \left\{k>0: \int_{\Omega} \Phi(\|f\| / k) d \mu \leqq 1\right\}$ or, if

1 The results announced here are contained in the author's doctoral thesis written under the guidance of Professor M. M. Rao at Carnegie Institute of Technology. 
$\mu$ has the finite subset property, $\operatorname{FSP}(A \in \Sigma, \mu(A)=\infty$, only if there is $B \in \Sigma, B \subset A, 0<\mu(B)<\infty),\|f\|_{\Phi}=\sup \left\{\int_{\Omega}\|f\|\|g\| d \mu, g \in L^{\Psi}\left(\mathfrak{X}^{*}\right)\right.$, $\left.N_{\Psi}(g) \leqq 1\right\} . M^{\Phi}(\mathfrak{X}) \subset L^{\Phi}(\mathfrak{X})$ is the closed subspace determined by the $\mu$-simple functions. If $\Phi$ satisfies the $\Delta_{2}$ condition $\left(\Phi(2 x) \leqq K^{\Phi}(x)\right)$, $M^{\Phi}(\mathfrak{X})=L^{\Phi}(\mathfrak{X}) \cdot L^{\Phi}(\mathfrak{X})$ and $V^{\Phi}(\mathfrak{X})$ are related by

Theorem 1. Let $\Phi$ be continuous, for $f \in L^{\Phi}(\mathfrak{X})$, define $\lambda f$ by $\lambda f(E)$ $=\int_{E} f d \mu, E \in \Sigma_{0}$. The mapping $\lambda$ maps $L^{\Phi}(\mathfrak{X})$ linearly into $V^{\Phi}(\mathfrak{X})$ and $N_{\Phi}(f)=N_{\Phi}(\lambda f)$. If $\mu$ has FSP and $f \in M^{\Phi}(\mathfrak{X}),\|f\|_{\Phi}=\|\lambda f\|_{\Phi}$.

2. The structure of $V^{\Phi}(\mathfrak{X})$. When $\Phi(x)=|x|$, the corresponding $V^{\Phi}(\mathfrak{X})$ is denoted by $V^{1}(\mathfrak{X})$ and is endowed with the variation norm $\mathfrak{v}(\cdot)$. The study of the structure of $V^{\Phi}(\mathfrak{X})$ rests upon the following generalization of the Radon-Nikodym-Bochner theorem [4, IV.9].

Theorem 2. Let $\mu(\Omega)<\infty$ and $F \in V^{1}(\mathfrak{X})$. If

$$
\left\{\frac{F(E)}{\mu(E)}:\left\|\frac{F(E)}{\mu(E)}\right\| \leqq n, E \in \Phi\right\}
$$

is weakly sequentially compact for each positive integer $n$, then for each $\epsilon>0$, there exists a $\mu$-simple function $f_{\epsilon}$ such that $\mathfrak{v}\left(F-\lambda f_{\epsilon}\right)<\epsilon$ where $\lambda$ is the injection of Theorem 1.

For $F \in V^{\Phi}(\mathfrak{X})$ and each partition $\pi=\left\{E_{n}\right\}, F_{\pi}$ is defined by

$$
F_{\pi}=\sum_{\pi} \frac{F\left(E_{n}\right)}{\mu\left(E_{n}\right)} \mu .
$$

$E_{n}$ where $\mu \cdot E_{n}$ is the set function defined by $\mu \cdot E_{n}(E)=\mu\left(E_{n} \cap E\right)$, $E \in \Sigma_{0} . S^{\Phi}(\mathfrak{X})$ denotes the closed subspace of $V^{\Phi}(\mathfrak{X})$ of functions satisfying $\lim _{\pi} N_{\Phi}\left(F-F_{\pi}\right)=0$ where the limit is taken in the Moore-Smith sense.

THEOREM 3. If $\Phi$ obeys the $\Delta_{2}$ condition and $\mathfrak{X}$ is reflexive $S^{\Phi}(\mathfrak{X})$ $=V^{\Phi}(\mathfrak{X})$.

3. Linear operators on $V^{\Phi}(\mathfrak{X})$ and $L^{\Phi}(\mathfrak{X}) . B(\mathfrak{X}, \mathfrak{Y})$ denotes the $B$ space of bounded linear operators from $\mathfrak{X}$ to $\mathfrak{Y}$.

Definition. $W^{\Phi}(\Omega, \Sigma, \mu, B(\mathfrak{X}, \mathfrak{Y}))=\left(W^{\Phi}(B(\mathfrak{X}, \mathfrak{Y}))\right.$ consists of all finitely additive $\mu$-continuous $B(\mathfrak{X}, \mathfrak{Y})$-valued set functions $H$ defined on $\Sigma_{0}$ and satisfying (i) $y^{*} H \in V^{\Phi}\left(\mathfrak{X}^{*}\right)$ for all $y^{*} \in \mathfrak{Y}^{*}$ and (ii) $\sup _{|| \nu^{*}|| \leq 1} N_{\Phi}\left(y^{*} H\right)=\|H\|_{W^{\Phi}<\infty}$.

Theorem 4. Let $\Phi$ be continuous. Then

(a) to each $h \in B\left(S^{\Phi}(\mathfrak{X}), \mathfrak{Y}\right)$ there corresponds a unique $H$ $\in W^{\Psi}(B(\mathfrak{X}, \mathfrak{V}))$ such that 


$$
h(F)=\lim _{\pi} \sum_{\pi} \frac{H\left(E_{n}\right)\left[F\left(E_{n}\right)\right]}{\mu\left(E_{n}\right)}, \quad F \in S^{\Phi}(\mathfrak{X}) .
$$

(b) If $S^{\Phi}(\mathfrak{X})$ is normed with $\|\cdot\|_{\Phi}$, the correspondence $h \rightarrow H$ maps $B\left(S^{\Phi}(\mathfrak{X}), \mathfrak{Y}\right)$ isometrically isomorphically onto $W^{\mathbb{\Psi}}(B(\mathfrak{X}, \mathfrak{Y}))$.

Since the injection $\lambda$ of Theorem 1 maps $M^{\Phi}(\mathfrak{X})$ onto a dense subset of $S^{\Phi}(\mathfrak{X})$, it follows that $B\left(M^{\Phi}(\mathfrak{X}), \mathfrak{Y}\right)$ is equivalent to $W^{\Psi}(B(\mathfrak{X}, \mathfrak{V}))$ with the representation of $h \in B\left(M^{\Phi}(\mathfrak{X}), \mathfrak{D}\right)$ taking the form

$$
h(f)=\lim _{\pi} \sum_{\pi} \frac{H\left(E_{n}\right)\left[\int_{E_{n}} f d \mu\right]}{\mu\left(E_{n}\right)}, \quad f \in M^{\Phi}(\mathfrak{X}), \quad H \in W^{\mathbb{\Psi}}(B(\mathfrak{X}, \mathfrak{V})) .
$$

4. Martingales. Here generalizations of the classical conditional expectation operator and martingales to the $V^{\Phi}(\mathfrak{X})$ setting are given.

Definition. Let $\Phi$ obey the $\Delta_{\mathbf{2}}$-condition and $B$ be a subfield of $\Sigma$. For $F \in S^{\Phi}(\mathfrak{X}), P_{B}(F)$ is defined by $P_{B}(F)=\lim _{\pi_{B}} F_{\pi_{B}}$ where the limit is taken in the $V^{\Phi}(\mathfrak{X})$ topology through all partitions $\pi_{B} \subset B$.

$P_{B}$ and $E^{B}$, the usual conditional expectation operator [10] are intimately related. In fact if $\Sigma$ is a $\sigma$-field, $B$ is a sub $\sigma$-field of $\Sigma$ and $\mu$ is countably additive and finite on $\Sigma$, then $\lambda E^{B}(f)=P_{B}(\lambda f)$ for all $f \in L^{1}(\mathfrak{X})$ where $\lambda$ is the injection of $L^{1}(\mathfrak{X})$ in to $V^{1}(\mathfrak{X})$ of Theorem 1.

Definitron. Let $\Phi$ obey the $\Delta_{2}$-condition and $\left\{B_{\tau}, \tau \in T\right\}$ be an increasing net of subfields of $\Sigma$. $\left\{F_{\tau}, B_{\tau}, \tau \in T\right\}$ is an $S^{\Phi}(\mathfrak{X})$-martingale if $P_{B_{\tau_{1}}}\left(F_{\tau_{2}}\right)=F_{\tau_{1}}$ for $\tau_{2} \geqq \tau_{1}$.

Typical of the class of mean martingale convergence theorems which can be proved is

THEOREM 5. Let $\mathfrak{X}$ be reflexive, $\Phi$ obey the $\Delta_{\mathbf{2}}$-condition and $\Psi$ be continuous. If $\left\{F_{\tau}, B_{\tau}, \tau \in T\right\}$ is an $S^{\Phi}(\mathfrak{X})$-martingale, then the net $\left\{F_{\tau}, \tau \in T\right\}$ converges in $N_{\Phi}(\cdot)$ norm if and only if there exists $P, 0<P<\infty$ such that $N_{\Phi}\left(F_{\tau}\right) \leqq P, \tau \in T$.

The following corollary which extends [3, Theorem 3$]$ is immediate from the properties of $\lambda$.

CoROLlaRY 7. Let $\Sigma$ be a $\sigma$-field and $\mu$ be countably additive and finite on $\Sigma$. If $\Phi$ obeys the $\Delta_{2}$-condition and $\Psi$ is continuous, a martingale $\left\{f_{\tau}, B_{\tau}, \tau \in T\right\}$ in $L^{\Phi}(\mathfrak{X})$ converges in $L^{\Phi}(\mathfrak{X})$ norm if and only if there exists $P, 0<P<\infty$ such that $N_{\Phi}\left(f_{\tau}\right) \leqq P, \tau \in T$.

\section{REFERENCES}

1. W. M. Bogdanowicz, Integral representation of linear continuous operators from the space of Lebesgue-Bochner summable functions into any Banach space, Proc. Nat. Acad. Sci. U. S. A. 54 (1965), 351-353. 
2. S. Bochner, Additive set functions on groups, Ann. of Math. 40 (1939), 769-799.

3. S. D. Chatterji, A note on the convergence of Banach-space valued martingales, Math. Ann. 153 (1964), 142-149.

4. N. Dunford and J. T. Schwartz, Linear operators, Part I, Interscience, New York, 1958.

5. K. Krickeberg and C. Pauc, Martingales et derivation, Bull. Soc. Math. France 91 (1965), 455-544.

6. S. Leader, The theory of $L^{p}$ spaces for finitely additive set functions, Ann. of Math. 58 (1953), 528-543.

7. R. S. Phillips, On weakly compact subsets of a Banach space, Amer. J. Math. 65 (1943), 108-136.

8. - On linear transformations, Trans. Amer. Math. Soc. 2 (1940), 516-541.

9. M. M. Rao, Linear functionals on Orlicz spaces, Nieuw Arch. Wisk. 12 (1964), $77-98$.

10. F. S. Scalora, Abstract martingale convergence theorems, Pacific J. Math. 11 (1961), 347-374.

11. A. Zygmund, Trigonometric series, 2nd ed., Cambridge Univ. Press, New York, 1959.

Carnegie Institute of Technology 